\title{
To Assess the Knowledge Regarding Signs and Symptoms of Diabetic Ketoacidosis and Its Prevention among Diabetes Patients in Wardha District, Maharashtra, India
}

\author{
Pratiksha Suresh Thakare'1, Ruchira Ankar² \\ ${ }_{1,2}$ Department of Medical Surgical Nursing, Smt. Radhikabai Meghe Memorial College of Nursing, Datta \\ Meghe Institute of Medical Sciences, Sawangi (M), Wardha, Maharashtra, India.
}

\section{ABSTRACT}

\section{BACKGROUND}

Diabetic ketoacidosis (DKA) is a life-threatening condition of diabetes that is characterized by hyperglycaemia, ketoacidosis, and ketonuria. It happens when glucose's ability to enter cells for use as metabolic fuel is blocked by absolute or relative insulin deficiency. This causes liver to divide fat into ketones as a source of fuel. The purpose of the study was to assess the knowledge regarding prevention of signs and symptoms of diabetic ketoacidosis among diabetes patients in selected hospitals of Wardha district.

\section{METHODS}

A descriptive study was undertaken among 60 purposively selected patients of diabetes with diabetic ketoacidosis in Wardha district. Data was collected by using structured knowledge questionnaire during the month of May 2020.

\section{RESULTS}

The level of awareness among diabetes patients on how to avoid diabetic ketoacidosis was measured. The findings of study show that $49(80 \%)$ people had poor level of knowledge score and 11 (20\%) people had average level of knowledge score. Mean score was $4.38 \pm 2.48$ with mean percentage $24.35 \pm 13.80$. The association of knowledge score with educational status of diabetes patients from selected hospital. The tabulated ' $\mathrm{F}$ ' values were $2.76(\mathrm{df}=3,56)$ which is much less than the calculated ' $F$ ' i.e., 8.73 at $5 \%$ level of significance. Also, the calculated ' $P$ ' $=0.0001$ which was much less than the acceptable level of significance i.e., ' $\mathrm{P}$ ' $=0.05$. Hence, it is interpreted that educational status of diabetes patients is statistically associated with their knowledge score.

\section{CONCLUSIONS}

Most patients with diabetes are poorly aware of the preventive measures of diabetic ketoacidosis. This study revealed several preventive gaps relating to an appropriate prevention strategy and acute diabetes complications. This research concluded that most patients with diabetes had insufficient knowledge of the signs and symptoms of diabetic ketoacidosis and its management.

\section{KEY WORDS}

Knowledge, Prevention, Sign, Symptoms, Diabetic Ketoacidosis, Diabetes Patients
Corresponding Author: Pratiksha Suresh Thakare, M.Sc. (Nursing) final year, Department of Medical Surgical Nursing, Smt. Radhikabai Meghe Memorial College of Nursing, Datta Meghe Institute of Medical Sciences, Sawangi (M), Wardha, Maharashtra, India.

E-mail: thakarep40@gmail.com

DOI: $10.14260 /$ jemds/2021/298

How to Cite This Article:

Thakare PS, Ankar R. To assess the knowledge regarding signs and symptoms of diabetic ketoacidosis and its prevention among diabetes patients in Wardha district, Maharashtra, India. J Evolution Med Dent Sci 2021;10(19):1413-1416, DOI: 10.14260/jemds/2021/298

Submission 01-12-2020,

Peer Review 09-03-2021,

Acceptance 16-03-2021,

Published 10-05-2021.

Copyright (c) 2021 Pratiksha Suresh Thakare et al. This is an open access article distributed under Creative Commons Attribution License [Attribution 4.0 International (CC BY 4.0)] 


\section{BACKGROUND}

Diabetic ketoacidosis is a life-threatening condition of diabetes that is characterized by hyperglycaemia, ketoacidosis, and ketonuria. It happens when glucose's ability to enter cells for use as metabolic fuel is blocked by absolute or relative insulin deficiency. This causes liver to divide fat into ketones as a source of fuel. Overproduction of ketones means that they accumulate in the blood and urine, turning the blood acidic in nature. DKA occurs typically in patients with type 1 diabetes, although it is not rare in some patients with type 2 diabetes. Glucose blood tests, serum electrolyte, blood urea nitrogen (BUN) and arterial blood gas are used in laboratory research for DKA. Treatment includes intravenous fluid loss correction, insulin hyperglycaemia correction, electrolyte disruption correction, particularly potassium loss, acid - base balance correction and the management of concomitant (if present) infections. ${ }^{1}$

Diabetes mellitus is a category of metabolic disorders characterized by elevated body glucose levels (hyperglycaemia) caused by insulin secretion defects, insulin activity, or both. In diabetes, the cells may stop fully responding to insulin. Long-term symptoms of hyperglycaemia lead to chronic micro - vascular complications (coronary artery disease, stroke, peripheral vascular obstructive disease), chronic micro - vascular complications (kidney and eye disease), and neuropathic complications (nervous diseases). ${ }^{2}$

In most of the type 1 diabetes patients, DKA was present. This type of diabetes is associated with the islets of Langerhans' in that absolute lack of insulin production is there. Insulin production is present in type 2 diabetes but is inadequate as a consequence of end - organ insulin resistance to satisfy the requirements of the body. These levels of insulin are usually enough to inhibit ketogenesis. Their disorder is called "ketosis - prone type 2 diabetes" if DKA exists in someone with type 2 diabetes mellitus. The particular mechanism for this condition is unknown, but its proof that insulin secretion and insulin action have been affected. If the disease has been controlled, the development of insulin resumes and, as usually prescribed in type 2 diabetes, the patient may also be able to resume diet. ${ }^{2}$

In those considered to have diabetes, DKA attacks can be avoided to a degree by agreeing with "sick day rules". The specific guidelines for patients about how to handle self-health condition while ill. Guidelines provide orders on how abundant extra insulin should be taken when sugar levels appear not in controlled level, an easily digestible diet rich in salt and carbohydrates, that means it helps in suppressing fever and managing further infection, and advice (such as a licensed diabetes teacher) when calling for medical aid. ${ }^{3}$

In the treatment of acute episodes of diabetes mellitus, such as hypoglycaemia, hyperglycaemia, or extreme ketoacidosis, the medical therapy and experience of health care professionals are most efficient. Majority of these episodes could be prevented by proper education and training of patients. Active involvement of the patient in the everyday treatment of diabetes tends to be the only viable solution for managing the condition and its long-term complications. ${ }^{4} \mathrm{An}$ acute important life - threatening complication of diabetes is diabetic ketoacidosis (DKA). DKA usually occurs in patients with type 1 diabetes, although in some patients with type 2 diabetes, it is not unusual. DKA is clinically described as an acute state of extreme ketoacidosis - associated uncontrolled diabetes that needs emergency treatment with insulin and intravenous fluids. ${ }^{1}$

Increasingly, diabetes is a big public health concern worldwide. Its influence is especially felt in developing countries, particularly in the regions of the Middle East and North Africa. To minimize the number of people living with diabetes mellitus worldwide, preventive steps should be taken. Sub - Saharan Africa is reported to be one of the regions with the fastest growing rates of diabetes mellitus in the world. Diabetic ketoacidosis (DKA) is an acute complication of diabetes. It is a metabolic emergency in individuals with absolute or relative insulin deficiency and is typically characterized by hyperglycaemia, ketonemia and acidosis. DKA was first described by Dreschfeld in 1886. The mortality rate of this disease was almost 100 percent before insulin was detected in 1922. There has been no decline in DKA mortality rates over the past 20 years. Around one third of DKA cases occur in newly diagnosed DM patients. Most deaths are caused by cerebral oedema complications. Awareness forms the basis for healthy health - related practices being implemented. The foundations of our future healthcare system are young people and adults, and it is important to assess and update their knowledge and understanding of diabetes and diabetic ketoacidosis. $^{5}$

A research by Zhong et al. found that in England, there was a rising incidence of hospitalization for DKA between 1998 and 2013 for adults' population with type 1 or type 2 diabetes mellitus. More precisely authors stated that an incidence of type 1 diabetes in patients increased between 1998 and 2007 and remained at the same level until 2013, while the incidence of type 2 diabetes increased by 4.24 percent annually between 1998 and 2013.6 The fourth leading cause of death from diseases worldwide is diabetes. In low - or middle - income countries, where many children with type I diabetes die because they lack access to life - saving insulin and where many do not obtain the requisite education to postpone and avoid complications, the burden is especially extreme. ${ }^{7}$ During researcher's clinical experience, it was found that diabetes mellitus patients having complications and many problems due to lack of knowledge, affect the quality of life of diabetes mellitus patient. Hence the researcher identified that an education to the care givers is necessary and selected this topic. ${ }^{8}$ As nurses play a crucial role in the treatment and control of patients with diabetes, sufficient information about the prevention of diabetic foot ulcers should be available. Nurses should inform patients about different diabetes risks and their preventive steps. Patients were also required to have self - care management information for the prevention of diabetic complications. ${ }^{9}$

\section{Objectives}

1. To assess the level of knowledge of diabetes patient regarding prevention of signs and symptoms of diabetes ketoacidosis.

2. To associate the knowledge of diabetes patients regarding prevention of signs and symptoms of diabetic ketoacidosis with selected demographic variables. 


\section{METHODS}

A descriptive study was undertaken on 60 purposively selected diabetic ketoacidosis among diabetes patients in Wardha district. Data was collected by using structured knowledge questionnaire from May 2020 to June 2020. Before the data collection, the willing consent from participant was taken in their understanding language. Those who were diagnosed with diabetic ketoacidosis were included in the study and those who already attended similar type of study were excluded in the study. A questionnaire was created in the most commonly spoken Marathi languages in the area. Participation was voluntary at the report. Participants answered the questionnaire themselves anonymously.

A tool was derived through several steps of item generation, reduction, weightage, pilot testing of the tool and validating of the tool. The content validity was determined after the opinion of 10 experts in the field of medical - surgical nursing. Tool validation included measurement of inter observer reliability; and generation of criterion related, construct related and content related validity. The tool was later revised by English - language expert and then translated into Marathi language by language experts without altering the tools meaning.

The Institutional Ethical committee of Datta Meghe, Institute of Medical sciences, deemed to be university sanctioned approval for conducting the research study Central Ethics Committee on Human Research. Ref. No. DMIMS (DU) / IEC / Dec - 2019 / 8642 was obtained. The findings were summarized by concentration and percentages categorically. The program used in the study was SPSS 24.0 and edition of graph pad prism 7.0 and $\mathrm{P}<0.05$ is regarded as a degree of significance. The questionnaire consisted of parts for acquiring demographic information and relevant questions related to the knowledge regarding prevention of signs and symptoms of diabetic ketoacidosis. A home - based analytical guide for prevention of signs and symptoms of diabetic ketoacidosis treatment was given.

To accomplish the objective of study, the investigator collected data from 60 diabetes patients by a self - prepared questionnaire including 18 questions based on prevention of signs and symptoms of diabetic ketoacidosis. Each correct answer carried 1 mark and 0 was given for the wrong response. Knowledge was graded from poor to good knowledge based on scores. The minimum score was (1) and maximum score was (18). Based on the total number of correct responses the degree of knowledge was classified as Poor (1 6), Average (7 - 12) and good (13 - 18).

\section{Statistical Analysis}

Descriptive and inferential statistics was used for analysis of data. The basic characteristics of the data were defined in descriptive statistics in a sample, and inferential statistics were used to draw inferences from our data to more general conditions. A percentage-wise distribution of diabetic ketoacidosis patient's demographic characteristics was determined. The statistical measures used for the study of the outcome were: Students unpaired $t$ - measure, one - way analysis of variance (ANOVA), Pearson's correlation coefficient and reliability analysis. By using Guttman Split Half method of reliability, it is found to be 0.9909 for knowledge and hence tool is reliable and valid. An appropriate sample was taken from the research population of 60 subjects at the Wardha district hospital. The data was gathered to identify sample characteristics such as age, gender, educational status, duration of disease.

\section{RESULTS}

Distribution of diabetic ketoacidosis patients according to their age in years shows that $6(10 \%)$ of them belonged to the age group of $20-30,12(20 \%)$ were in the age group of 31 $40,17(28.3 \%)$ were in the age group of $41-50$ and 25 (41.7 $\%)$ were in the age group of $51-60$.

\begin{tabular}{|c|c|c|c|}
\hline \multicolumn{2}{|c|}{ Demographic Characteristics. } & Subjects & Percentage \\
\hline \multirow{4}{*}{ Age (years) } & $20-30$ & 6 & 10.0 \\
\hline & $31-40$ & 12 & 20.0 \\
\hline & $41-50$ & 17 & 28.3 \\
\hline & $51-60$ & 25 & 41.7 \\
\hline \multirow{3}{*}{ Gender } & Male & 39 & 65.0 \\
\hline & Female & 21 & 35.0 \\
\hline & Primary & 28 & 46.7 \\
\hline \multirow{4}{*}{ Educational status } & Illiterate & 5 & 8.3 \\
\hline & Secondary & 22 & 36.7 \\
\hline & Graduate and above & 5 & 8.3 \\
\hline & $1-6$ months & 13 & 21.7 \\
\hline \multirow{3}{*}{ Duration of disease } & $7-12$ months & 21 & 35.0 \\
\hline & $13-18$ months & 20 & 33.3 \\
\hline & $>18$ months & 6 & 10.0 \\
\hline \multicolumn{4}{|c|}{$\begin{array}{c}\text { Table 1. Percentage Wise Distribution of Diabetic Ketoacidosis } \\
\text { Patients According to Their Demographic Characteristics }\end{array}$} \\
\hline
\end{tabular}

Distribution of patients according to their sex reveals that $39(65 \%)$ of them were male and 21 (35\%) were female. Distribution of diabetic ketoacidosis patients according to their educational qualifications shows that $28(46.7 \%)$ of them were educated up to primary, 5(8.3\%) of them were illiterate, $22(36.7 \%)$ of them were educated up to secondary, 5 ( $8.3 \%)$ of them were educated up to graduate and above.

Distribution of diabetic ketoacidosis patients according to their duration of disease, shows that $13(21.7 \%)$ of them were diagnosed for 1 - 6 months, 21(35\%) of them were diagnosed for 7 - 12 months, 20 (33.3\%) of them were diagnosed for 13 - 18 months, and 6 (10\%) of them were diagnosed since above 18 months. In assessment of patients, the level of knowledge score shows that $49(80 \%)$ patients were having poor level of knowledge score, $11(20 \%)$ had average level of knowledge score and $0 \%$ had a good level of knowledge score. The range of knowledge score was in between 1 - 11. Mean knowledge score was $4.38 \pm 2.48$ and the mean percentage of knowledge score was $24.35 \pm 13.80$.

Associating knowledge score with educational status of diabetic ketoacidosis patients from selected hospital. In this study the only educational status associated with knowledge score for comparing the knowledge score with education, an unpaired $t$-test was used. The tabulated ' $F$ ' values were 2.76 $(\mathrm{df}=3,56)$ which is much less than the calculated ' $\mathrm{F}$ ' i.e., 8.73 at $5 \%$ level of significance. Also, the calculated ' $P$ ' $=0.0001$ which was much less than the acceptable level of significance i.e., ' $\mathrm{P}$ ' $=0.05$. Hence, it is interpreted that educational status of diabetes patients is statistically associated with their knowledge score. 


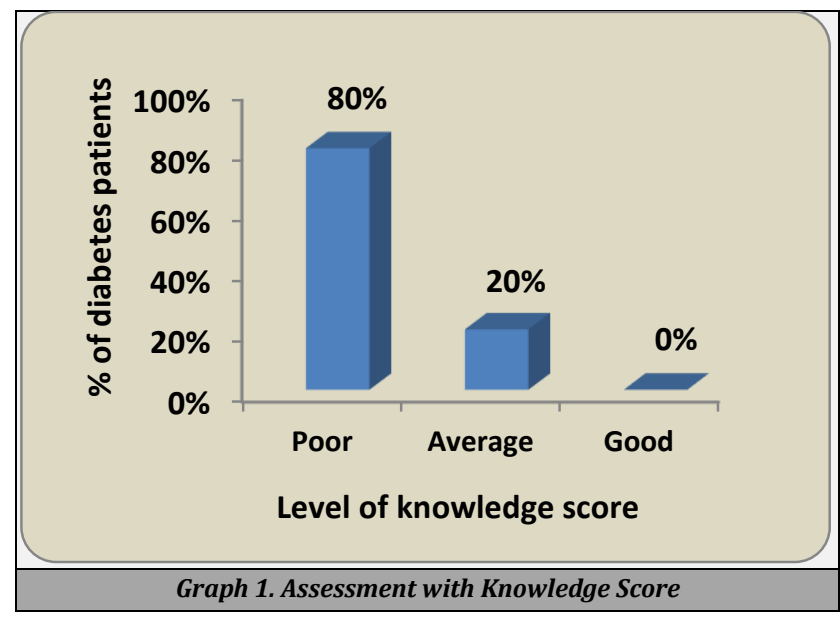

\section{DISCUSSION}

The study was conducted to determine the level of knowledge of DKA among diabetic patients in the Riyadh population, according to previous research. 150 samples have been obtained for analysis. The findings of the study indicate that 58 (38.67\%) participants had poor awareness of complications, 101 (67.34\%) had poor management knowledge, 9 (6.0\%) participants had good knowledge, 57 (38\%) participants had poor risk factor knowledge, and 45 (30\%) of participants had good awareness. The bulk of the respondents had a low level of understanding. A connection exists between having a first degree relative with diabetic mellitus and the level of DKA awareness. ${ }^{10}$

A similar research was undertaken to assess practitioners' and final-year medical students' experience and understanding of diabetic ketoacidosis (DKA). From October to December 2018, 82 practitioners and 302 medical students were gathered for study. According to the study's results, 72.5 percent was of diabetic ketoacidosis knowledge and just 39 percent was of diabetic ketoacidosis perception. In about $42 \%$ of questions about DKA awareness, there were substantial variations between the 2 groups. In these disparities, practitioners outperformed students in half of the questions, while students outperformed practitioners in the other half. In $(75 \%)$ of the questions, there were substantial gaps between the two classes in terms of DKA sensitivity, with practitioners showing higher awareness than students. With regard to the time of practice, there were no substantial differences in expertise and understanding among practitioners.

According to the results, there was inadequate degree of information and understanding about DKA in certain regions.

In my present analysis, the overall outcome was found to be low (80 percent) with questions about DKA awareness. In terms of priorities and theoretical basis, the results of the analysis were discussed.

The present study showed that $4.38 \pm 2.48$ was the total mean information score. $80 \%$ of diabetic patients have a poor knowledge score and $20 \%$ of diabetic patients have an average knowledge score about the prevention of diabetic ketoacidosis signs and symptoms.

Lack of information about DKA (80 percent) was seen among diabetes patients. In this research, educational status was significant.

\section{CONCLUSIONS}

Most patients with diabetes are poorly aware of the preventive measures of diabetic ketoacidosis. This study revealed several preventive gaps relating to an appropriate prevention strategy and acute diabetes complications. This research concluded that most patients with diabetes had insufficient knowledge of the signs and symptoms of diabetic ketoacidosis and its management. Small sample size is a limitation of this study.

Data sharing statement provided by the authors is available with the full text of this article at jemds.com.

Financial or other competing interests: None.

Disclosure forms provided by the authors are available with the full text of this article at jemds.com.

I would like to show my appreciation to Ms.Ruchira Ankar as my thesis Guide. Beyond that I would like to express my sincere thanks to all faculties of Department of Medical surgical Nursing for completion of my research work.

\section{REFERENCES}

[1] Hamdy 0, Khardori R. Diabetic Ketoacidosis (DKA): practice essentials, background, pathophysiology. Updated Jan 19, 2021. https://emedicine.staging.medscape.com/article/11836 1-overview

[2] Basavanthappa BT. Medical surgical nursing. $1^{\text {st }}$ edn. New Delhi: Jaypee Brother's Medical Publishers 2003: p. 687711.

[3] Basavanthappa BT. Nursing research. $1^{\text {st }}$ edn. New Delhi: Jaypee Brother's Medical Publishers 2005: p. 49.

[4] Patricia BG, Judith ML. Adult health nursing. $3^{\text {rd }}$ edn. Philadelphia: Mosby Publishers 1998: p. 1406-46.

[5] Ahmed HOM. Knowledge and awareness of diabetes and diabetic ketoacidosis among practitioners and final medical students in Wad Medani Gezira State, Sudan (2018). Thesis. University of Gezira, 2019 (cited on Jun 20, 2020). http://repo.uofg.edu.sd/handle/123456789/3630

[6] Zhong VW, Juhaeri J, Mayer-Davis EJ. Trends in hospital admission for diabetic ketoacidosis in adults with type 1 and type 2 diabetes in england 1998-2013: a retrospective cohort study. Diabetes Care 2018;41(9):1870-77.

[7] Joyce B, Jane H. Medical Surgical Nursing. $7^{\text {th }}$ edn. Missouri: Saunders's Publishers 2005: p. 1243-88.

[8] Gail HH, Judith DR. Total patient care. $8^{\text {th }}$ edn. Philadelphia: Mosby Publishers 1992: p. 595-611.

[9] Suresh S. Nursing research and statistics. $2^{\text {nd }}$ edn. India: Elsevier Health Sciences 2014.

[10] Farran BA, Elaiwah RIB, Aldarsouny AT, et al. Level of awareness of diabetic ketoacidosis among diabetes mellitus patients in Riyadh. J Family Med Prim Care 2020;9(6):2676-9. 